\title{
An Atypical Presentation of 'Possible' Neurosarcoidosis: A Stepwise Approach to a Case with Review of Literature
}

\author{
Bhanu Gogia ${ }^{1^{*}}$, Varun B. Shah ${ }^{1}$, Prashant K. Rai ${ }^{1}$, Karthikram Raghuram ${ }^{2}$ and Chilvana Patel ${ }^{1}$ \\ ${ }^{1}$ Department of Neurology, University of Texas Medical Branch, Galveston, TX, USA \\ ${ }^{2}$ Department of Neuroradiology, University of Texas Medical Branch, Galveston, TX, USA
}

Correspondence to:

Dr. Bhanu Gogia, MD

Neurology Resident Physician

University of Texas Medical Branch at Galveston 301 University Blvd, Galveston

TX 77555, United States

E-mail: bhgogia@utmb.edu

Received: February 19, 2020

Accepted: May 26, 2020

Published: May 27, 2020

Citation: Gogia B, Shah VB, Rai PK, Raghuram K, Patel C. 2020. An Atypical Presentation of 'Possible' Neurosarcoidosis: A Stepwise Approach to a Case with Review of Literature. J Neuroimaging Psychiatry Neurol 5(1): 16-19.

Copyright: (C) 2020 Gogia et al. This is an Open Access article distributed under the terms of the Creative Commons Attribution 4.0 International License (CC-BY) (http://creativecommons. org/licenses/by/4.0/) which permits commercial use, including reproduction, adaptation, and distribution of the article provided the original author and source are credited.

Published by United Scientific Group

\begin{abstract}
Objective: To report an atypical case that highlights the challenges involved in diagnosing neurosarcoidosis.

Case description: A 38-year-old Caucasian man presented with a three month history of headache and a ten day history of confusion and abnormal movements in the right upper extremity. The patient did not have any other systemic symptoms such as visual disturbances, arthritis, oral or genital ulcers, dry eyes or dry skin, but reported a history of weight loss, night sweats as well.MR brain showed extensive white matter disease with deep grey matter involvement, linear perivascular enhancement and deep medullary venous engorgement, suggesting deep vein thrombosis. Cerebrospinal fluid (CSF) labs revealed a leukocytosis of 98/ul, glucose of $39 \mathrm{mg} / \mathrm{dl}$, and protein of $413 \mathrm{mg} / \mathrm{dl}$. CSF angiotensin converting enzyme (ACE) positive at $4 \mathrm{U} / \mathrm{L}$. The infectious and rheumatological workup were negative including fungal cultures, MTB PCR, Quant Gold test, ANA, Anti SSA, SSB, ANCA, HIV, HBV. Computed tomography (CT) chest showed pulmonary granulomatous disease and calcified hilar lymphadenopathy. Meningeal biopsy was negative for granulomas. After considering and ruling out conditions such as Behcet's disease, Sjogren's, vasculitis, and CNS lymphoma. Our patient fitted into 'possible' neurosarcoidosis due to a lack of pathological confirmation of granulomatous disease.
\end{abstract}

Conclusion: Diagnosis of neurosarcoidosis remains challenging with the multitude of symptoms and non-specific laboratory tests. Broad differentials including neoplasm especially lymphoma, infectious etiologies such as fungal, mycobacterium and alternate inflammatory conditions including Behcet's should be considered and excluded appropriately. Neurosarcoidosis is one of the common inflammatory conditions associated with cerebral venous sinus thrombosis.

\section{Keywords}

Neurosarcoidosis, Neuroinflammation, Deep medullar venous engorgement, Deep cerebral venous thrombosis, White matter disease, Secondary headache

\section{Introduction}

Neurosarcoidosis is an inflammatory disease that occurs in about 5\%-10\% of cases of patients with sarcoidosis [1]. Neurosarcoidosis is known for its heterogeneity in regards to its clinical manifestations and is often challenging to diagnose $[1,2]$. From a meta-analysis done in 2016, neurosarcoidosis is reported to be slightly more common in females than males with a propensity towards 
African American people [1]. The clinical manifestation is highly variable, most commonly being cranial neuropathies [3]. The neuroimaging findings of neurosarcoidosis described in the literature include engorgement of the deep medullary veins, leptomeningeal or dural disease, perivascular nodular enhancement, venous thrombosis, hydrocephalus, and hemorrhage [4-6]. Cerebral venous thrombosis, although rare but has been reported in neurosarcoidosis, where hypercoagulability is presumably due to active granulomatous inflammation with an increased concentration of inflammatory markers [7]. We report an atypical case that highlights the challenges involved in diagnosing neurosarcoidosis that will enhance the readers' knowledge and help them contemplate beyond traditional diagnoses.

\section{Methods}

We searched PubMed for 'neurosarcoidosis', 'neuroinflammation', 'neuroimaging in neurosarcoidosis', 'cerebral venous sinus thrombosis'.

\section{Discussion}

\section{Case description}

A 38-year-old right-handed caucasian man with no past medical history presented with a three- month history of headache and a ten-day history of confusion and abnormal movements in the right upper extremity. At the onset of his headache, he had an extensive workup done at an outside hospital, which was unremarkable. He also endorsed significant weight loss and night sweats during this period. Of note, he was planning a trip to Africa and had multiple immunizations, including Yellow fever, $\mathrm{TDaP}$ (Tetanus, Diphtheria, acellular Pertussis), and MMR (Measles, Mumps, and Rubella) prior to his symptom onset. Over the past ten days, he had increased somnolence and thus presented to our hospital. The headache was described as pressure type, 10/10 severity, worsened with light but no phonophobia, no positional change and no associated vision change. Due to concern for a secondary headache syndrome, brain imaging including magnetic resonance imaging (MRI) brain with and without contrast and MR Venogram were obtained. MR brain (Figure 1) showed extensive white matter disease with deep grey matter involvement, linear perivascular enhancement and deep medullary venous engorgement with no diffusion restriction suggesting an inflammatory process along with deep vein thrombosis. MRI brain findings were suggestive of CNS inflammatory process such as neurosarcoidosis and less likely to be CNS lymphoma. MR Venogram confirmed thrombosis of the left-sided internal cerebral vein, left sigmoid sinus and the vein of trolard partly seen in figure 2. Considering the degree of white matter involvement, the venous thrombosis of the deep medullary vein was thought to be a secondary phenomenon due to an inflammatory process.

Additionally, the hypercoagulable work up with protein $\mathrm{C}$, S, Anti b2 glycoprotein, Anti cardiolipin Ab, Homocysteine level were within normal limits and Factor $\mathrm{V}$ and Factor

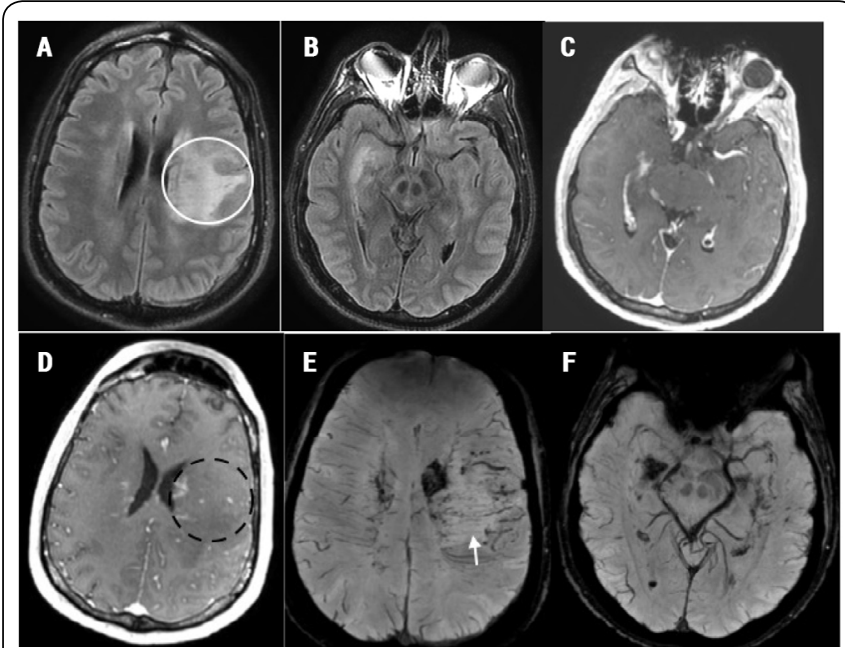

Figure 1: Figure shows different sequences of magnetic resonance imaging (MRI) brain at the time of presentation. MR T2 FLAIR (A and B) that show hyperintensity in left frontoparietal subcortical white matter and cortical grey matter (white circle), MR T1 with contrast (C-D) showing patchy area of enhancement (black circle), susceptibility weighted images (SWI) (E and $\mathbf{F})$ showing deep medullary venous engorgement.

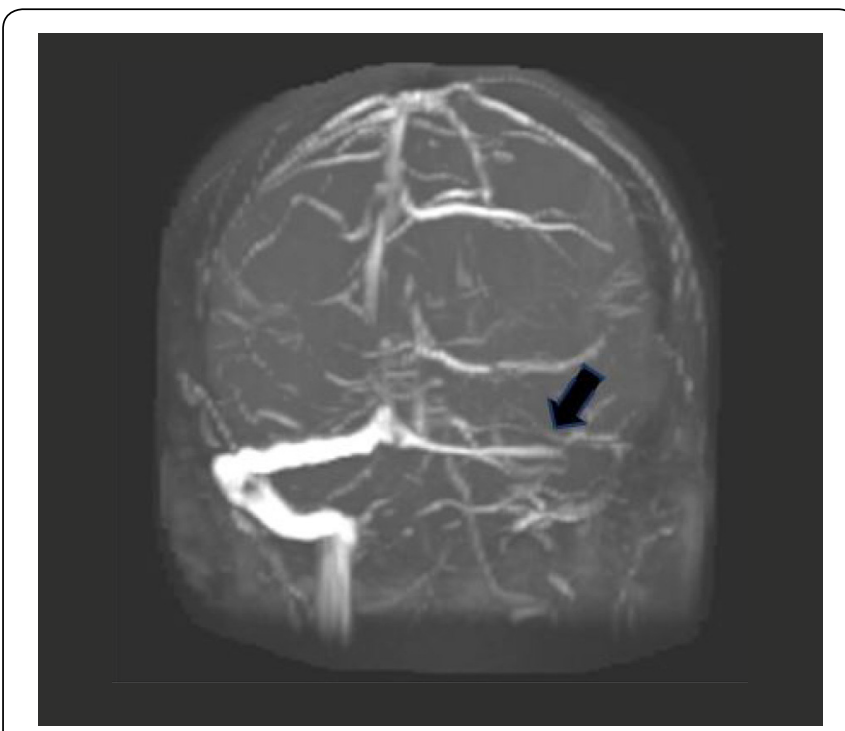

Figure 2: MR venogram with a black arrow pointing at lack of flow in the left sigmoid sinus and jugular bulb along with some irregularity in the left transverse sinus suggesting venous sinus thrombosis.

G20210A resulted negative. The rheumatological workup including ANA,AntiSSA,SSB,ANCAwas negative.Following the imaging studies, lumbar puncture (LP) was performed. Opening pressure was $165 \mathrm{~cm} \mathrm{H}_{2} \mathrm{O}$ and CSF labs revealed a WBC of 98/ul with lymphocytic predominance, glucose of $39 \mathrm{mg} / \mathrm{dl}$, and protein of $413 \mathrm{mg} / \mathrm{dl}$ with positive oligoclonal bands (OCBs). Infectious etiologies such as tuberculosis, histoplasmosis, coccidioidomycosis, blastomycoses were ruled out in serum as well as CSF. Cytology was negative, but flow cytometry showed monoclonal plasma cells raising suspicion for lymphoma. Electroencephalogram (EEG) showed only focal slowing in the left hemisphere. As the cytology was negative and the flow cytometry was non-confirmatory, an MR spectroscopy of brain was performed, which was inconclusive. Later, positive OCBs favored inflammatory etiology, and 
positive $\mathrm{CSF}$ angiotensin converting enzyme (ACE) suggested a granulomatous disease like neurosarcoidosis. Because of the diagnostic dilemma, steroids were held until a brain biopsy could be performed. In the interim, a repeat LP performed after 6 days showed protein of $234 \mathrm{mg} / \mathrm{dl}$, glucose of $45 \mathrm{mg} /$ $\mathrm{dl}$, and WBC 41/ul (lymphocytic).

The patient deteriorated clinically, therefore intravenous dexamethasone was started, and the biopsy was postponed. Following three days on steroids, the patient improved clinically. Repeat MRI brain showed significant improvement in the white matter T2-hyperintensity. CSF cytology was negative the second time, but CSF ACE remained positive at 4 U/L. CT chest showed pulmonary granulomatous disease and calcified hilar lymphadenopathy. After considering and ruling out various autoimmune and infectious etiologies with the aforementioned laboratory testing, the diagnosis at that time was neurosarcoidosis, and less likely lymphoma. The patient was discharged on a steroid taper and apixaban for venous thrombosis. While on the steroid taper, patient had a bronchoscopy which showed lymphoid tissue but no malignant cells or granulomas. A month after discontinuation of steroids, the patient returned to our clinic for a follow-up visit and reported worsening of his headache. At that time, a repeat MRI brain (Figure 3) showed an improvement in confluent periventricular and deep cerebral white matter hyperintensity with linear perivascular enhancement suggesting neurosarcoidosis versus demyelinating lesion. Lumbar puncture revealed (WBC 17, protein 159mg/ $\mathrm{dl}$, glucose $39 \mathrm{mg} / \mathrm{dl}$ ) and elevated CSF ACE at $2.9 \mathrm{U} / \mathrm{L}$. Following this, the patient had a craniotomy with meningeal

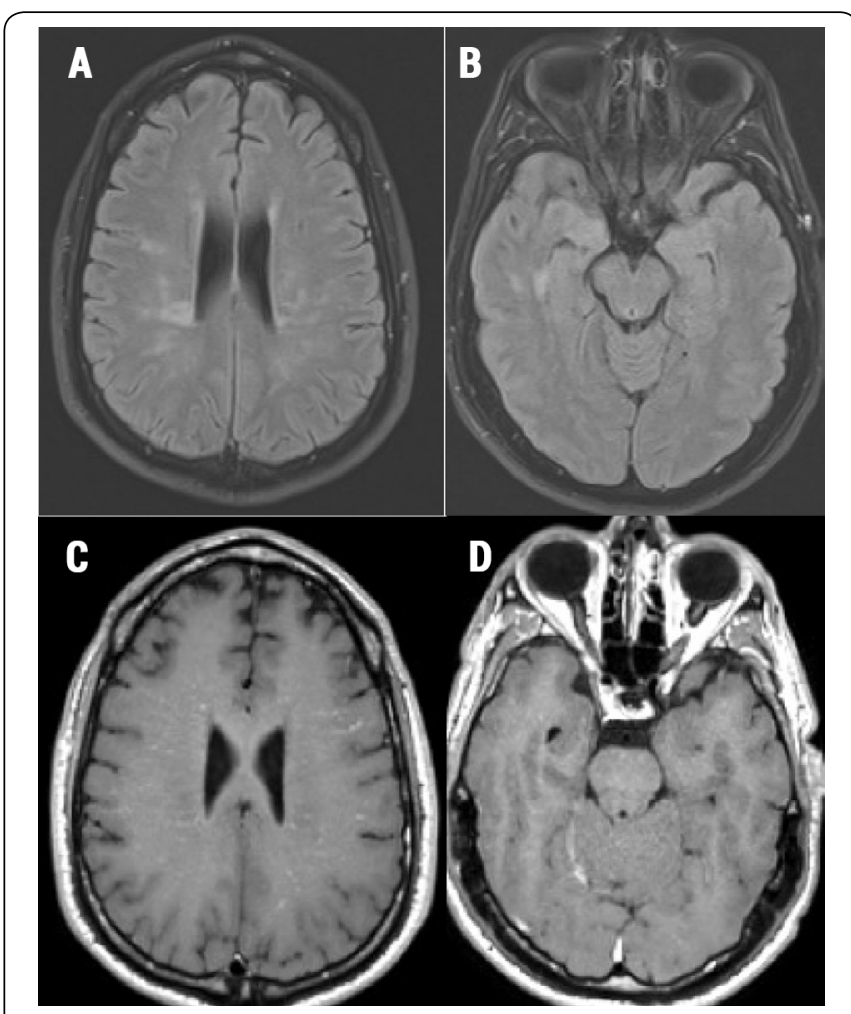

Figure 3: MR brain T2 FLAIR (A and $\mathbf{B}$ ) and T1 with contrast $(\mathbf{C}, \mathbf{D})$ images show improvement in periventricular and deep cerebral white matter hyperintensity with redemonstration of patchy enhancement bilaterally. resection, which showed no evidence of inflammation or neoplasm.

\section{Case discussion}

Neurosarcoidosis is known for its heterogeneity in regards to its clinical manifestations and is often challenging to diagnose $[1,2,5]$. From a meta-analysis done in 2016, neurosarcoidosis is reported to be slightly more common in females than males with a propensity towards African American people [3]. Since the patient described in this report is a caucasian male, neurosarcoidosis was not the top differentials. While making a diagnosis of neurosarcoidosis, the other differentials that need to be considered and ruled out are Behcet's disease, Sjogren's, vasculitis, multiple sclerosis, and malignancies. Our patient did not have any other systemic symptoms such as visual disturbances, arthritis, oral or genital ulcers, dry eyes or dry skin, and rheumatological workup was negative including Antinuclear Antibody (ANA), Anti SSA, SSB, Anti-Neutrophilic Cytoplasmic Autoantibody (ANCA), Human Immunodeficiency Virus (HIV), Hepatitis B Virus (HBV). Vasculitis and malignancy were also considered and ruled out on conventional angiogram (DSA) and CSF cytology and biopsy, respectively. In the majority of the cases, pulmonary sarcoidosis is present, but isolated neurosarcoidosis has been reported in $22 \%$ cases [3]. In this patient, the presence of ground-glass opacities and granulomatous pulmonary disease on CT chest in the presence of negative mycobacterium tuberculosis (MTB) PCR, quant gold test supported the diagnosis. Among the multitude of neurological signs and symptoms that can be seen, most common are cranial nerve palsies followed by headache and sensory abnormalities [4].

Our patient initially presented with headache followed by abnormal movement and then weakness in his right upper extremity with altered sensorium. The ancillary testing available such as angiotensin converting enzyme (ACE) has low sensitivity (24\%-55\%) and specificity (94\%-95\%) for an accurate diagnosis and leaving brain biopsy as the ultimate step, which has some serious implications [6, 7]. Due to its challenging diagnosis, there exist three diagnostic criteria Zajicek, World Association of Sarcoidosis and Other Granulomatous Diseases (WASOG), and the recently proposed diagnostic criteria by Stern et al. [8]. Based on the latest guidelines described by Stern et al., in 2018 [8], our patient fits into 'possible' neurosarcoidosis due to a lack of pathological confirmation of granulomatous disease. In our patient, he had MRI FLAIR (fluid attenuated inversion recovery) signal changes as well as CSF findings supporting the inflammatory process. Additionally, the presence of thrombosis in the deep medullary veins further supported an underlying inflammatory condition and imaging findings similar to what has been described in relation to neurosarcoidosis rather than other etiologies [9-12].

Currently, there exists no randomized trial to assess treatment strategies. The mainstay of treatment is corticosteroids. However, other steroid-sparing agents such as methotrexate, azathioprine, leflunomide, mycophenolate, and infliximab are increasingly used in current practice, especially for maintenance therapy [13]. 


\section{Conclusion}

Diagnosis of neurosarcoidosis can be very challenging with the multitude of symptoms, non-specific laboratory tests, and imaging findings. It is one of the common inflammatory conditions associated with cerebral venous sinus thrombosis with neuroimaging findings similar to what is described in this paper. The case described in this report also highlights the challenges and utility of current guidelines into clinical practice.

\section{Conflict of Interest}

The authors declare no conflict of interest.

\section{References}

1. Aksamit A. 2008. Neurosarcoidosis: neurologic manifestations of systemic disease. Continuum 14 (1):181-196. http://doi.org/10.1212/01. CON.0000299992.09447.2b

2. Tavee JO, Stern BJ. 2014. Neurosarcoidosis. Continuum (Minneap Minn) 20(3 Neurology of Systemic Disease): 545-559. https://doi. org/10.1212/01.con.0000450965.30710.e9

3. Fritz D, van de Beek D, Brouwer MC. 2016. Clinical features, treatment and outcome in neurosarcoidosis: systematic review and meta-analysis. BMC Neurol 16(1): 220. https://doi.org/10.1186/s12883-016-0741-x

4. Fritz D, Voortman M, van de Beek D, Drent M, Brouwer MC. 2017. Many faces of neurosarcoidosis: from chronic meningitis to myelopathy. Curr Opin Pulm Med 23(5): 439-446. https://doi.org/10.1097/ mcp.0000000000000401

5. Voortman M, Drent M, Baughman RP. 2019. Management of neurosarcoidosis: a clinical challenge. Curr Opin Neurol 32(3): 475-483. https://doi.org/10.1097/wco.0000000000000684
6. Ungprasert P, Carmona EM, Crowson CS, Matteson EL. 2016. Diagnostic utility of angiotensin-converting enzyme in sarcoidosis: a population-based study. Lung 194(1): 91-95. https://doi.org/10.1007/ s00408-015-9826-3

7. Khoury J, Wellik KE, Demaerschalk BM, Wingerchuk DM. 2009. Cerebrospinal fluid angiotensin-converting enzyme for diagnosis of central nervous system sarcoidosis. Neurologist 15(2): 108-111. https:// doi.org/10.1097/nrl.0b013e31819bcf84

8. Stern BJ, Royal W, Gelfand JM, Clifford DB, Tavee J, et al. 2018. Definition and consensus diagnostic criteria for neurosarcoidosis: from the neurosarcoidosis consortium consensus group. JAMA Neurol 75(12): 1546-1553. http://doi.org/10.1001/jamaneurol.2018.2295

9. Bathla G, Watal P, Gupta S, Nagpal P, Mohan S, et al. Cerebrovascular manifestations of neurosarcoidosis: an underrecognized aspect of the imaging system. AJNR Am J Neuroradiol 39(7): 1194-1200. https://doi. org/10.3174/ajnr.a5492

10. Zamora C, Hung SC, Tomingas C, Atkinson C, Castillo M. 2018. Engorgement of deep medullary veins in neurosarcoidosis: a commonyet-underrecognized cerebrovascular finding on SWI. AJNR Am J Neuroradiol 39(11): 2045-2050. https://doi.org/10.3174/ajnr.a5783

11. Goljan-Geremek A, Geremek M, Puscinska E, Sliwinski P. 2015. Venous thromboembolism and sarcoidosis: co-incidence or coexistence? Cent Eur J Immunol 40(4): 477-480. https://doi.org/10.5114/ ceji.2015.56972

12. Haldorsen IS, Espeland A, Larsson EM. 2011. Central nervous system lymphoma: characteristic findings on traditional and advanced imaging. AJNR Am J Neuroradiol 32(6): 984-992. https://doi.org/10.3174/ajnr. a2171

13. Flanagan EP. 2019. Neuromyelitis optica spectrum disorder and other non-multiple sclerosis central nervous system inflammatory diseases. Continuum (Minneap Minn) 25(3): 815-844. https://doi.org/10.1212/ con.0000000000000742 